\author{
Suárez Saá, Beatriz \\ Investigadora de la Universidad de Vigo (UVIGO), Facultad de Bellas Artes de Pontevedra (FBAP), \\ Grupo de Investigación (PS1).
}

\title{
Entre lo humano y el entorno. Los nuevos híbridos.
}

\author{
TIPO DE TRABAJO \\ Comunicación virtual. \\ PALABRAS CLAVE \\ Arte, híbrido, frontera, orgánico, tecnológico.
}

KEY WORDS

Art, hybrid, border, organic, technology.

\section{RESUMEN}

Vivimos en la era de lo tecnológico. El ser humano ha cambiado sus hábitos de vida y el modo en el que se relaciona con el entorno en las últimas décadas. Los nuevos aparatos tecnológicos nos rodean e invaden por donde quiera que vamos, y se han vuelto "indispensables" en muestra sociedad global; hasta el punto de convertirse en extensión de nuestros cuerpos, e incluso podríamos decir, que en "prótesis" de los mismos. Los límites entre lo humano y el entorno se han empezado a desdibujar a una velocidad atroz y hasta límites inconcebibles. Lo real y lo virtual se confunden. Las fronteras se han esfumado y esto se ha observado directamente en el arte y en sus nuevas producciones. Y es que el siglo XXI se puede considerar la época de la hibridación, tanto a nivel conceptual, de definición identitaria, como a nivel de formalización, y más concretamente, en las recientes manifestaciones artísticas. Este texto tratará de hacer una revisión conceptual y visual de algunas de las producciones artísticas de nuestros días, en las que se muestran estos conceptos.

\section{ABSTRACT}

We live in an age of technological. Humans have changed their lifestyle and the way it relates to the environment in recent decades. The new technological devices around us and invade wherever they go, and have become "indispensable" to show global society; to the point of becoming extension of our bodies, and we might even say that in "prosthesis" of them. The boundaries between the human and the environment have begun to blur a terrible speed and to inconceivable limits. The real and the virtual are confused. The borders are gone and this has been directly observed in art and new productions. And the twenty-first century can be considered the time of hybridization, both conceptually, defining identity, and at the level of formalization, and more specifically, in recent artistic expressions. This text will try to make a conceptual and visual review of some of the artistic productions of our time, in which these items are.

\section{CONTENIDO}

Introducción

Voy a empezar este pequeño texto, planteándole la cuestión que yo misma me cuestioné antes de comenzar a escribir las líneas que dan forma al mismo. ¿Qué es un híbrido? Deténgase por un instante, piénselo, y dese una rápida respuesta. Intente quedarse con esas primeras impresiones latentes en su cabeza, mientras a través de mis reflexiones trato de desengranar, muy por encima, el concepto, sin perder de vista el contexto artístico que nos compete. 
Si seguimos la definición que de esta palabra se da en el diccionario de la real academia española ${ }^{1}$, podemos citar tres descripciones de este adjetivo. La primera: "dicho de un animal o de un vegetal que es procreado por dos individuos de distinta especie". La segunda: "dicho de un individuo cuyos padres son genéticamente distintos con respecto a un mismo carácter". Y la tercera: "se dice de todo lo que es producto de elementos de distinta naturaleza".

Dicho así parece que lo híbrido no tiene una relación completamente directa con nosotros. En la primera, nos quedamos aparentemente fuera, observándolo en nuestros vecinos, los animales y las plantas. Se sugiere, en la segunda, que se puede dar la circunstancia, en casos determinados, de que entorno a lo humano se permite generar algo híbrido. Y la tercera, resulta tan abierta, que dependiendo de lo que se defina como distinta naturaleza, casi todo puede serlo, pero tampoco se concreta.

$\mathrm{Mi}$ apreciación al respecto, es que lo híbrido está íntegramente presente en nosotros y en todo nuestro entorno, sobre todo en las últimas décadas, años de gran avance técnico, y en cuyo campo considero que se muestra esto de una forma rotunda. El arte de por sí, es un territorio naturalmente heterogéneo, ya que mezcla lenguajes de distinta naturaleza que dialogan a la par, dando lugar a lo que podríamos denominar géneros o manifestaciones híbridas; $u$ obras, como las definimos a diario. ¿Pero no es, al fin y al cabo esto, una descripción del ser humano? ¿No es el hombre en sí, un híbrido, por encima de todo? ¿No somos seres compuestos por múltiples "mecanismos", fragmentarios, que percibimos y nos relacionamos con los "otros", a través de pedazos y composiciones hechas de los mismos? ¿A través, de conjuntos de lenguajes, percibidos por los sentidos, que nuestras mentes luego fusionan e interpretan? El pasado siglo trajo consigo numerosos avances en múltiples campos del conocimiento y a nivel social, pero si cabe destacar alguno en concreto, deberíamos decir que el siglo XX fue la centena de años, a lo largo de la historia de la humanidad, de más progresos a nivel tecnológico ${ }^{2}$.

Vivimos, actualmente, en esta era. El ser humano ha cambiado sus hábitos de vida y el modo en el que se relaciona con el entorno. Los nuevos aparatos mecánicos nos rodean e invaden por donde quiera que vamos, y se han vuelto "indispensables" en muestra sociedad global; hasta el punto de convertirse en extensión de nuestros cuerpos, e incluso podríamos decir, que en "prótesis" de los mismos, siendo solo a veces, conscientes de ello. Los límites entre lo humano y el entorno se han empezado a desdibujar a una velocidad atroz y hasta límites inconcebibles. Lo real y lo virtual se confunden. Las fronteras se han esfumado y esto se ha observado directamente en el arte y en sus nuevas producciones. Y es que el siglo XXI se puede considerar la época de la hibridación, tanto a nivel conceptual, de definición identitaria, como a nivel de formalización, y más concretamente, en las recientes manifestaciones artísticas.

La fotografía y el cine abrieron la veda a nuevas posibilidades el pasado centenario, e Internet y la utilización de los nuevos medios informáticos, lo han empezado a explotar llegando a extremos inconcebibles. Muchos artistas de nuestro tiempo investigan y reflexionan sobre esta cuestión, llegando a confundir ese límite entre lo real y lo ficticio, y en correlación, con lo virtual. El simulacro se explota al extremo y el espectador es inundado por la duda de lo que es veraz y de lo que es fingido. Los híbridos de artistas consagrados como Matthew Barney, Patricia Piccinini, Joan Fontcuberta; los ciborgs de Marina Núñez, Stelarc...; las esculturas hiperrealistas de Xooang Choi y Ron Mueck; las performances de Orlan..., entre otros; y las producciones de jóvenes creadores como María Castellanos, Estela de Frutos...; serán citados para hacer un pequeño recorrido sobre esta cuestión tan amplia. Al mismo tiempo, quiero señalar, que estas clasificaciones las he hecho simplemente para organizar los conceptos a nivel textual, ya que posiblemente muchos de sus creadores no lo hubieran indicado así, y solo desde este punto vista, pretendo que se perciba.

\section{Desarrollo}

Entrando ya en materia, dentro del terreno de los híbridos, quiero resaltar los que se distinguen como tal, a nivel formal, por ser o tener una mezcla de elementos de cuerpos provenientes de distintas naturalezas (animal, vegetal y humana), conviviendo entre sí. Los primeros ejemplos de ello de los que quiero hablar, son los creados por el artista y video creador estadounidense Matthew Barney $(1967)^{3}$, y más concretamente, en dos de los personajes que aparecen en su Ciclo Cremaster, (obra a medio camino entre el cine y el vídeo-arte, compuesto por cinco películas, esculturas, fotografías, dibujos y el cuaderno del propio artista), realizado entre el año 1994 y el 2002. Y más concretamente, dentro del mismo, en la parte fílmica, en la mujer-guepardo, presente en Cremaster 3 (2002), y en el fauno con dos pares de cuernos, que aparece en Cremaster 4 (1994), e interpretado por el propio artista. A continuación añado dos fotografías de los mismos, en las que se pueden observar como el cuerpo humano y el animal se fusionan de forma armoniosa para dar lugar a otro tipo de "seres", en este caso ficticios, aunque la barrera entre lo real y lo "virtual", debido al gran realismo en su representación, intenta, de alguna manera, confundirnos. No obstante, este es un papel recurrente del cine, por lo que quizás, en este caso, el engaño puede resultar menor.

1 “híbrido", Diccionario de la lengua española (22a edición), Real Academia Española (2001), consultado el 28 de abril de 2015.

2 Carvalho, M. Híbridos tecnológicos. Lisboa: Ed. Nova Vega, 2007, ISBN 978-972-699-839-6.

3 Matthew, B. Cremaster [en línea]. <www.cremaster.net> [Consulta: el 27 de Abril de 2015] 

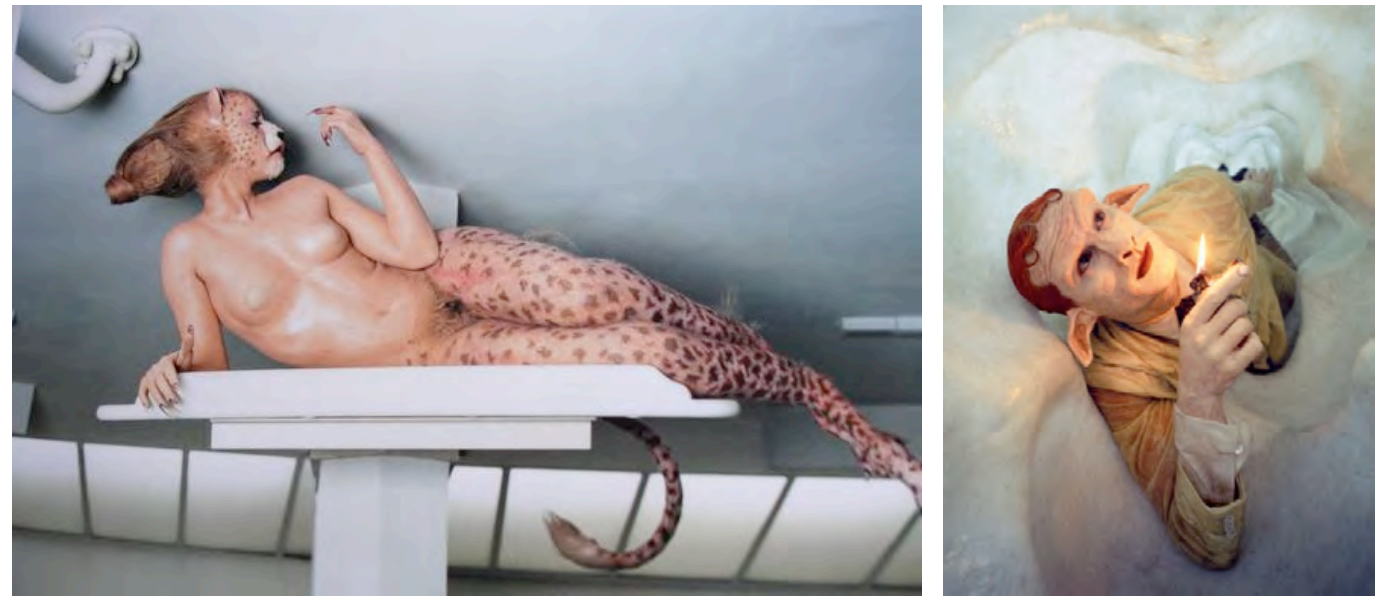

Imagen 1. Mujer-guepardo, Fotograma perteneciente a la película Cremaster 3, 2002, Mattew Barney Imagen 2. Fauno con cuernos, Fotograma perteneciente a Cremaster 4, 1994, Matthew Barney

Cremaster es una obra de una gran complejidad a nivel formal y conceptual. Posee continuas reminiscencias a la sexualidad humana, concretamente al músculo cremáster y a la posición de los órganos reproductivos durante el proceso de la diferenciación sexual, a las etapas de desarrollo fetal, a las mutaciones genéticas...; todo ello influencia del campo de la medicina en el que se formó el artista antes de dedicarse a la creación, y que forman parte de su universo simbólico junto con otros rasgos narrativos provenientes de su biografía, de la mitología, y la arquitectura, entre otros. Y sus personajes no son más que la conjunción de estos aspectos aunque a una menor escala.

Otra creadora, nada en Sierra Leona en 1965, ésta ya en el campo de la escultura realista, o más bien "hiperrealista", Patricia Piccinini ${ }^{4}$, trabaja desde sus inicios con el mismo concepto, aunque en ella se percibe de una forma mucho más clara e intencionada. Usando materiales como las resinas, siliconas, pelo humano...; crea obras tridimensionales cuyos protagonistas parecen extraídos de nuestra imaginación, o del mundo onírico infantil. Resultado también de la simbiosis de los tres terrenos (animal, humano y vegetal) dentro de un mismo cuerpo, aunque en su caso bajo cierto aspecto mucho más dócil y amigable que en otros creadores; muchos de sus "personajes" desprenden un carácter tierno que nos transmite cercanía. Esto se nos muestra a través de formas grotescas, signos de lo "diferente" o de lo "raro naturalmente", pero siempre de una manera muy próxima y natural, como si estuvieran presentes a diario en nuestra cotidianeidad. En este caso lo diferente ya no es lejano, sino todo lo contrario. Y de hecho, esta cercanía creo que es fundamental para entender su trabajo, y por ello lo destaco. La frontera entre nosotros y esos "otros seres anormales" se desdibuja por completo, aspecto que se muestra claramente en las obras recogidas en las fotografías siguientes.



Imagen 3. The long awaited ${ }^{5}$, Patricia Piccinini, escultura, 2008

4 Piccinini, P. Patricia Piccinini [en línea]. <http://www.patriciapiccinini.net/ > [Consulta: 27 de Abril de 2015]

5 Roslyn Oxley 9 Gallery. Artists: Patricia Piccinini [en línea] <http://www.roslynoxley9.com.au/artists/31/Patricia_Piccinini/1124/41436/> [Consulta: 9 de Mayo de 2015] 
A su vez, quiero indicar, que la obra de Piccinini nos recuerda, por cierta similitud formal en lo que se refiere al potencial hiperrealista de sus obras, a las creaciones del australiano Ron Mueck $(1958)^{6}$, o a las del coreano Xooang Choi (1975) ${ }^{7}$. Ambos con un dominio formal y técnico espectacular_al igual que la africana_, desde el punto de vista de la recreación, o la similitud con lo humano, cada uno, eso sí, bajo su estilo propio y personal; siguen poniendo en entredicho, y de forma obvia, esa frontera entre lo real y lo ficticio. Mueck rompe esa tela de ficción jugando con la escala, y Choi resalta la incapacidad y el fracaso humano a través de lo absurdo y lo anómalo. Las siguientes obras de ambos, son algún ejemplo de ello.


Imagen 4. In bed, Ron Mueck, escultura, 2005

Imagen 5. Vegetative state, Xooang Choi, escultura, 2007

Y ya sobrepasando ese límite de la ficción y lo real, podemos destacar el trabajo del español Joan Fontcuberta (1955) ${ }^{8}$. Este creador nos involucra por completo en sus fotografías, sobre todo en la serie Fauna, en la que sus híbridos, en este caso, entre animales de distintas especies, parecen completamente reales, falseando ser nuevas especies descubiertas, y su fotografía el registro de las mismas a modo documental. Su labor se asemeja a la de un falso zoólogo que recoge muestras visuales de sus investigaciones y las difunde. Aquí juega no solo con el concepto de mezcla, sino también con el concepto del arte y la labor del artista y de su trabajo.

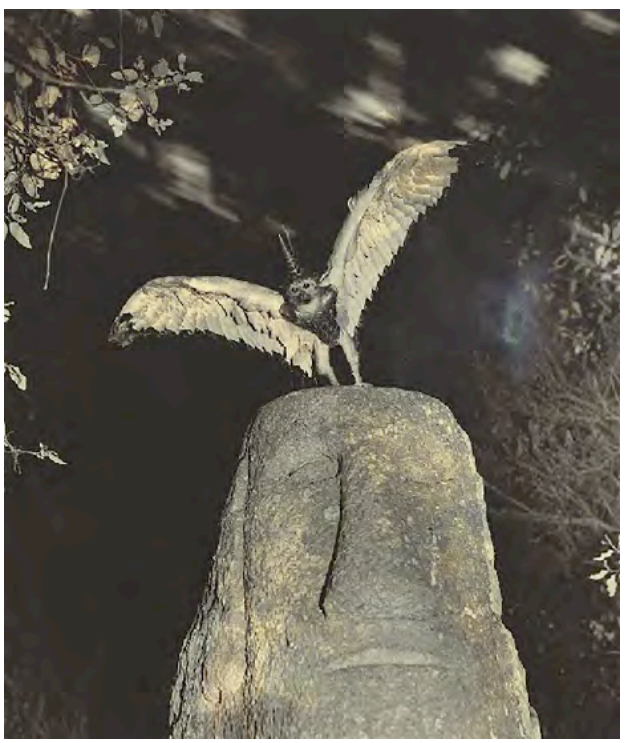

Imagen 6. Cercopithecus Icarocomu, Joan Fontcuberta, fotografía de la serie fauna, 1985

6 Brooklyn Museum. Exhibitions: Ron Mueck. [en línea] <https://www.brooklynmuseum.org/exhibitions/ron_mueck/in_bed.php> [Consulta: 29 de abril de 2015]

7 Dossan Gallery. Archive: Xooang Choi [en línea] <http://www.doosangallery.com/artists/works_eng.asp?idx=119\&workldx=1478\&subPageNum=17> [Consulta: 27 de Abril de 2015]

8 Fontcuberta, F. Joan Fontcuberta [en línea]. <http://www.fontcuberta.com/> [Consulta: 28 de Abril de 2015]. 
Y a medio camino entre una identidad híbrida, en diálogo continuo, sin frontera, de los tres campos que mencionaba anteriormente, y centrándonos también en el concepto de prótesis añadida al ser humano, quiero citar a la joven artista madrileña Estela de Frutos $(1983)^{9}$. Esta creadora multidisciplinar, recoge elementos naturales, inertes, provenientes del mundo vegetal y animal, y les otorga una "nueva vida" a través de sus intervenciones. Ejemplo de ello es la obra perteneciente a las serie Cuerpo germinal, en la que a través de la escultura y la fotografía al mismo tiempo, muestra la no frontera de territorios en lo humano y añade un enfoque de cuestionamiento existencial y espiritual que no está presente, o no tan claramente, en los artistas de los que ya he hablado.


Imagen 7. Querer volar, Estela de frutos, fotografía, 2012

Imagen 8. Cuerpo germinal, Estela de frutos, fotografía, 2012

Entra aquí en escena un elemento que es fundamental para entender al grupo de artistas que voy a citar a continuación, y este es, la prótesis, con sus diversas apreciaciones o usos. Aquí debo de nombrar en primer lugar a Stelarc ${ }^{10}$, Chipre (1946). Artista, principalmente performer, que integra la robótica y la tecnología directamente en el cuerpo. Trabaja con sistemas de realidad virtual, Internet, biotecnología... Y como un ejemplo de ello, he de señalar, la obra Third hand, en la que se instaló una tercera mano, un brazo "virtual", o Ern on arm, en la que se incorporó una oreja en el brazo, como muestras de su labor. Consiguiendo disolver, a través de sus personales fórmulas, las barreras entre lo mecánico y lo carnal. Y en relación directa con él, es imprescindible nombrar en este punto, a la artista francesa Orlan (1947) ${ }^{11}$, que justamente ha explorado el concepto de prótesis en una dirección similar al chipriota, pero en su caso, llegando a rozar límites que cortejan el delirio. Realizó a lo largo de su carrera diversas performances en las que llegó a someterse a múltiples operaciones quirúrgicas, en las que se cambió por completo sus rasgos físicos, faciales, perdiendo así, lo que podríamos definir, como sus rasgos identitarios personales. Ella, se sirve de los avances mecánicos para establecer juegos entre la desfiguración y la refiguración, aunque su planteamiento se podría enfocar concretamente desde los territorios que cuestionan la identidad, o aspectos de diferenciación de género, a diferencia de Stelarc.


Imagen 9. Amplified body, laser eyes and third hand, Stelarc ${ }^{12}$, Fotografía registro de la performance, 1985 Imagen 10. Operación acertada, Orlan, fotografía en Cibachrome, 1991, París.

9 De Frutos, E. Estela de Frutos [en línea]. <www.esteladefrutos.com> [Consulta: 28 de Abril de 2015].

10 Stelarc. Stelarc [en línea]. < http://stelarc.org/ > [Consulta: 6 de Mayo de 2015]

11 Orlan. Works: Performance. [en línea]. <www.orlan.eu > [Consulta: 8 de Mayo de 2015]

12 CEC, Canadian electroacoustic comunity. Gallery: Stelarc [en línea] Julio 2012. <http://cec.sonus.ca/econtact/14_2/stelarc_gallery.html> [Consulta: 9 de Mayo de 2015] 
Y llegados a este instante, quiero introducir aquí el concepto de cyborg, ya que la obra de los dos últimos artistas que he indicado, ya se podrían clasificar bajo este término definitorio. El cyborg no es más que un híbrido, pero en este caso concreto, "la conjunción es entre lo humano y la máquina, una criatura de realidad social y de ficción” ${ }^{13}$. Lo mecánico y lo tecnológico se muestran como extensión del cuerpo humano. Donna Haraway utiliza justamente el término, en su Manifiesto para Cyborg ${ }^{14}$ _texto que se considera el origen de los ciberfeminismos_, para definir la realidad social de la contemporaneidad, señalando, en el mismo, la desaparición de las divisiones entre lo orgánico y lo mecánico, lo material y lo inmaterial, entre las identidades sexuales ${ }^{15}$.

Como subraya Haraway, "las fonteras entre ciencia ficción y la realidad son ilusión óptica"16. Y justamente, en este punto de cuestionamientode la ficción y la realidad podemos incluir también la obra de la artista palentina Marina Núñez (1966)17. En la que, a través de sus series Cyborgs, o Ciencia ficción, platea y profundiza en muchas de las cuestiones que Haraway adelanta en su texto.

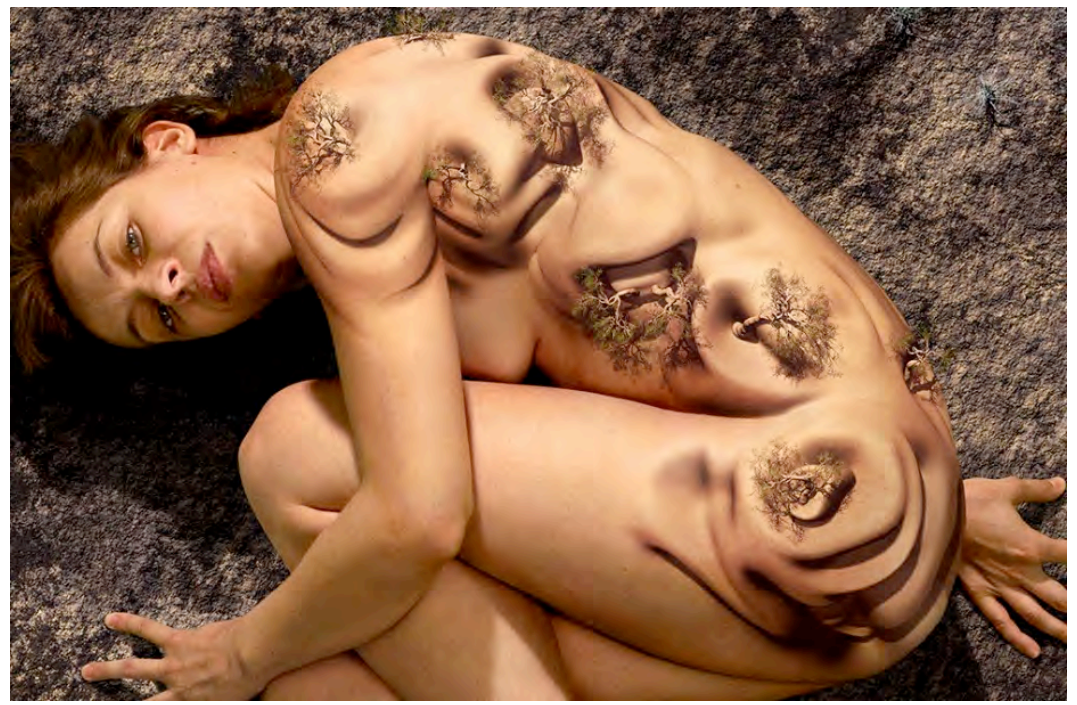

Imagen 11. Sin título (serie: ciencia ficción). Marina Núñez. Detalle. Infografía sobre tela, 2010.

Y en relación con esta, y ya para finalizar, me gustaría hablar de la obra de una joven creadora asturiana, María Castellanos (1985)18, que tiene un interesante trabajo en el que investiga acerca del uso continuo de las nuevas tecnologías en nuestro día a día. Muestra como las mismas influyen en nuestros cuerpos y nuestras formas de percibir o mirar, y como lo real y lo aparentemente invisible, entran en un diálogo paralelo, puesto en relieve, gracias a la presencia de los aparatos técnicos. En una de sus obras más conocidas, "Corpo-realidad", se presenta ella misma vistiéndose con un traje realizado con un material especial, que hace que la gente que la está viendo en directo la perciba de forma normal, pero al mismo tiempo, está siendo grabada un equipo de vídeo, que gracias al material del que está compuesto el vestido, permite que se capture lo invisible para los asistentes al acto. Y es que en la grabación se puede ver el cuerpo desnudo de María por debajo del mismo, en un determinado momento de la acción.

13 AACA, Asociación Aragonesa de Críticos de Arte. Ciborgs y mutantes [en línea]: lo humano y lo posthumano en la obra de Matrhew Barney, realizado por Monika keska. 30 de Julio de 2009. Revista online Estudios del Arte, no 7, Junio 2007, ISSN 1988-5180.

<http://www.aacadigital.com/contenido.php?idarticulo=212> [Consultada: 2 de Mayo de 2015].

14 Haraway, D. Manifiesto para cyborg. Ciencia, tecnología y feminismo socialista a finales del siglo XX, Valencia: Eutopía, 1985, ISSBN 8489055858. 15 Haraway, D. Manifiesto para cyborg. Ciencia, tecnología y feminismo socialista a finales del siglo XX, Valencia: Eutopía, 1985, pp. 3-5, ISSBN 8489055858

16 Haraway, D. 1985, Ibidem.

17 Nuñéz, M. Marina Núñez [en línea]. <www.marinanunez.net > [Consulta: 9 de Mayo de 2015]

18 Castellanos, M. María Castellanos Vicente [en línea]. < http://mariacastellanos.net/> [Consulta: 9 de Mayo de 2015] 

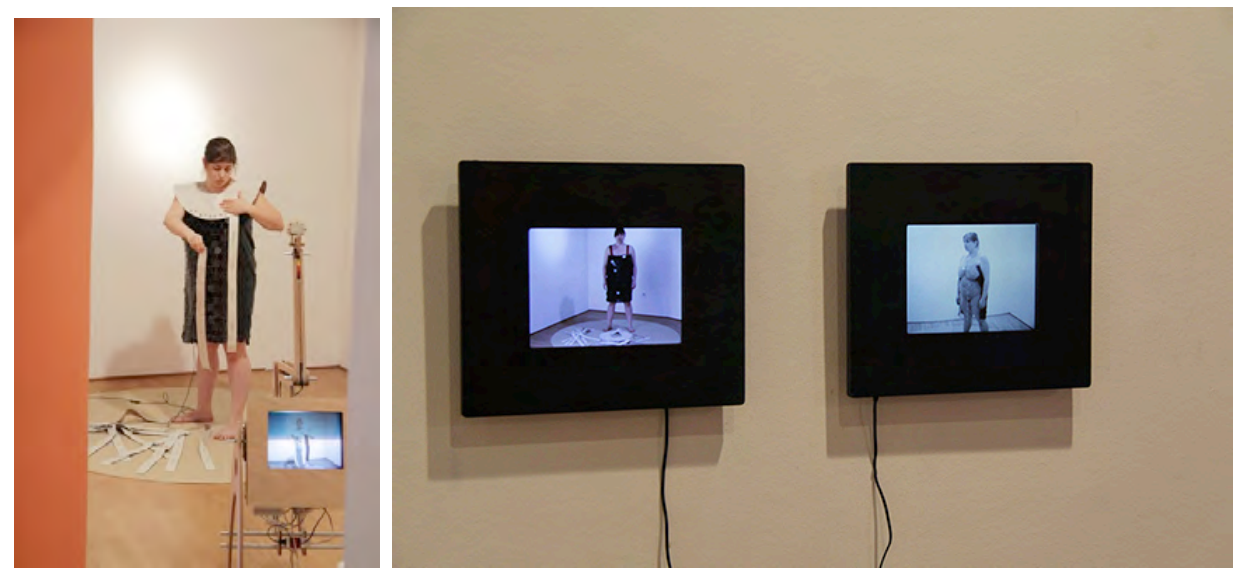

Imagen 12 . "Corpo-realidad", María Castellanos, Fotografías registro de performance en la Sala Astragal de Gijón, 2013.

\section{Conclusiones}

Lo híbrido es un reflejo de nuestro tiempo, símbolo de la postmodernidad y de la contemporaneidad, tal y como he indicado anteriormente, y como señalaba Haraway en su Manifiesto ${ }^{19}$. Pero híbrido no es un concepto nuevo, si no que siempre ha sido un aspecto que ha estado presente en la vida del ser humano, desde sus orígenes, aunque en la actualidad, arropado por circunstancias específicas que tienen que ver con la globalización, la presencia arrolladora de Internet y de los mass media en nuestras vidas, los avances tecnológicos y el consumo masivo de los mismos...; hacen que hoy éste tenga un papel protagonista y se pueda percibir de una forma más clara.

A su vez, son muchos los artistas que trabajan y reflexionan acerca de esta cuestión, tal y como he mostrado, ya que forma parte de sus existencias, aunque muchos de ellos no sean conscientes de que lo están haciendo, o no se definan bajo el concepto de híbrido con que yo los he acuñado. No obstante, como he mostrado, todos ellos presentan visiones muy particulares y diferentes del concepto, desde diversos campos creativos y con una gran riqueza definitoria. Pero en todos ellos, símbolos o productos de nuestro tiempo, se puede advertir lo que señalaba anteriormente, las fronteras, tanto de lo orgánico como de lo tecnológico, y el hecho de como las mismas se han desdibujado, como aquello que es real o es ficticio, o por correlación, virtual, conviven en nuestros días a la par. Nosotros no somos más que testigos o víctimas del engaño de su existencia. O lo que podría indicarse de otro modo, nosotros no somos más que los residuos de los mismos. Lo que Daniel Canogar, Madrid $(1964)^{20}$, muestra en sus intervenciones. Quizás el residuo sea ese el lugar hacia el que nos dirigimos, y todo ese compendio de restos que consumimos y acumulamos a un ritmo atroz desde hace unos años, sea lo que nos torture en el futuro, lo que nos "hibridice".

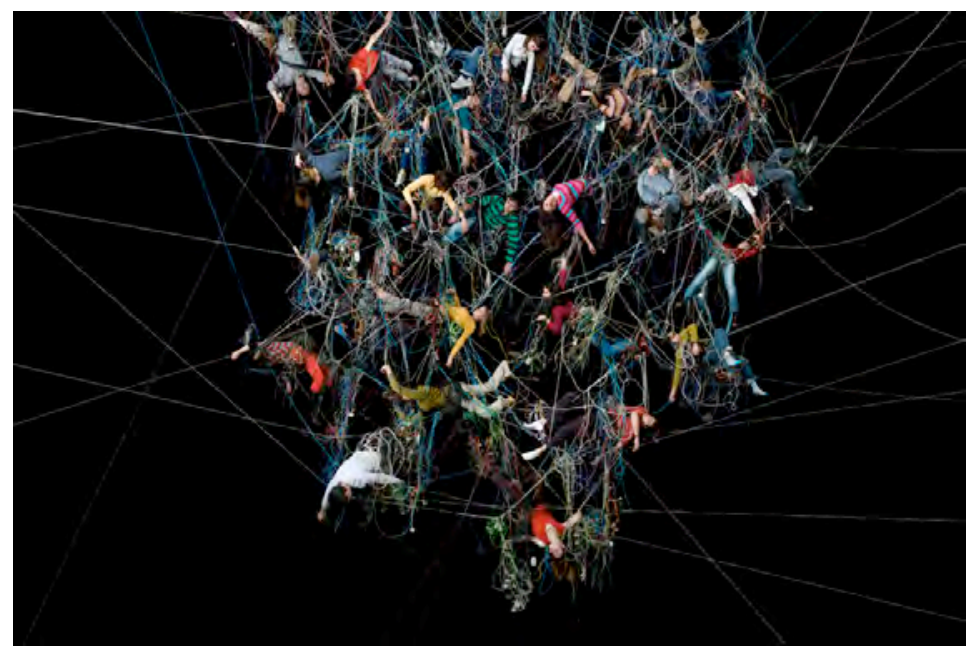

Imagen 13. Enredos, Daniel Canogar, fotografía digital impresa en kodak endura, 2008

19 Haraway, D. 1985. Vid.

20 Canogar, D. Daniel Canogar. [en línea]. <http://www.danielcanogar.com/?lang=es> [Consulta: 9 de Mayo de 2015] 


\section{FUENTES REFERENCIALES.}

\section{Bibliografía}

Carvalho, M. Híbridos tecnológicos. Lisboa: Ed. Nova Vega, 2007, ISBN 978-972-699-839-6.

Diccionario de la lengua española (22a edición), Real Academia Española (2001), consultado el 28 de abril de 2015.

Haraway, D. Manifiesto para cyborg. Ciencia, tecnología y feminismo socialista a finales del siglo XX, Valencia: Eutopía, 1985, ISSBN 8489055858 .

\section{Recursos electrónicos}

Matthew, B. Cremaster [en línea]. <www.cremaster.net> [Consulta: el 27 de Abril de 2015]

Piccinini, P. Patricia Piccinini [en línea]. <http://www.patriciapiccinini.net/ > [Consulta: 27 de Abril de 2015]

Roslyn Oxley 9 Gallery. Artists: Patricia Piccinini [en línea]

<http://www.roslynoxley9.com.au/artists/31/Patricia_Piccinini/1124/41436/> [Consulta: 9 de Mayo de 2015]

Brooklyn Museum. Exhibitions: Ron Mueck. [en línea] <https://www.brooklynmuseum.org/exhibitions/ron_mueck/in_bed.php> [Consulta: 29 de abril de 2015]

Dossan Gallery. Archive: Xooang Choi [en línea]

<http://www.doosangallery.com/artists/works_eng.asp?idx=119\&workldx=1478\&subPageNum=17 > [Consulta: 27 de Abril de 2015]

Fontcuberta, F. Joan Fontcuberta [en línea]. <http://www.fontcuberta.com/> [Consulta: 28 de Abril de 2015].

De Frutos, E. Estela de Frutos [en línea]. <www.esteladefrutos.com> [Consulta: 28 de Abril de 2015].

Stelarc. Stelarc [en línea]. < http://stelarc.org/ > [Consulta: 6 de Mayo de 2015]

Orlan. Works: Performance. [en línea]. <www.orlan.eu > [Consulta: 8 de Mayo de 2015]

AACA, Asociación Aragonesa de Críticos de Arte. Ciborgs y mutantes [en línea]: lo humano y lo posthumano en la obra de Matrhew Barney, realizado por Monika keska. 30 de Julio de 2009. Revista online Estudios del Arte, no 7, Junio 2007, ISSN 1988-5180. $<$ http://www.aacadigital.com/contenido.php?idarticulo=212> [Consultada: 2 de Mayo de 2015].

Nuñéz, M. Marina Núñez [en línea]. <www.marinanunez.net > [Consulta: 9 de Mayo de 2015]

Castellanos, M. María Castellanos Vicente [en línea]. < http://mariacastellanos.net/> [Consulta: 9 de Mayo de 2015

Canogar, D. Daniel Canogar. [en línea]. <http://www.danielcanogar.com/?lang=es> [Consulta: 9 de Mayo de 2015] 